\title{
Diseño Mecánico de una Plataforma Multi-registro para la Verificación de Instrumentos de Medir por Coordenadas Portátiles
}

\author{
Agustín Brau, Jorge Santolaria, Raquel Acero, Juan José Aguilar \\ GIFMA (Grupo de Ingeniería de Fabricación y Metrología Avanzada) \\ Instituto de Investigación en Ingeniería de Aragón (I3A). \\ Universidad de Zaragoza, Mariano Esquillor s/n, 50018, Zaragoza, Spain. \\ Tel. +34-976762707, Fax +34-976762043, e-mail: agusba2003@gmail.com
}

\begin{abstract}
En este trabajo se presenta el diseño de una plataforma multi-registro cuyo objetivo final es mejorar y simplificar los procedimientos de verificación e identificación de parámetros en instrumentos de medición por coordenadas portátiles. Además se muestran sus componentes y sistemas mecánicos que permiten su uso en dichos procedimientos.
\end{abstract}

\section{Introducción}

En la actualidad, los procesos de fabricación industrial tienen que satisfacer tolerancias de diseño cada vez más ajustadas. Esto, sumado a la gran automatización de los procesos en los últimos tiempos, hace indispensable contar con métodos y equipos de medida que permitan medir de forma rápida garantizando el cumplimiento de las tolerancias especificadas del producto En años recientes se ha incrementado considerablemente el uso de equipos de medida portátiles en los procesos industriales debido a su gran flexibilidad para realizar mediciones de gran complejidad y su bajo coste comparado con las máquinas de medir por coordenadas (MMC) tradicionales. Algunas desventajas que presentan los equipos portátiles tales como los brazos articulados de medir por coordenadas (BAMC) o laser trackers residen en las técnicas de procedimientos de verificación y optimización de parámetros [1], [2]. Éstas técnicas se basan en la captura de datos con el instrumento de medida de un objeto patrón calibrado, como por ejemplo una barra patrón de esferas o un tetraedro patrón de esferas, dispuesto en varias posiciones dentro del volumen de medida del equipo con el fin de cubrir gran parte de su espacio de trabajo. El hecho de colocar el patrón alrededor del instrumento de medida, hace del procedimiento de verificación un proceso tedioso y de excesiva duración, además de hacer necesario el uso de soportes que permitan posicionar de manera rígida el patrón en distintas alturas $\mathrm{y}$ orientaciones respecto al equipo a verificar. En vista de lo anterior, la simplificación y optimización de las técnicas de verificación e identificación de parámetros es un tema de actualidad e importancia en la literatura y la investigación en el área.

\section{Componentes mecánicos y mecanismos de la plataforma}

La plataforma multi-registro está compuesta por una placa fija o base (figura 1b) y una placa móvil (figura 1a) que gira sobre ella. La placa móvil utiliza un mecanismo de elevación manual que le permite girar cada $60^{\circ}$ con respecto a la placa fija, lo que hace posible colocar la placa móvil en 6 posiciones diferentes con respecto a la placa fija. Para referenciar cada una de estas posiciones se utilizan acoplamientos cinemáticos de cilindros y esferas de acero dispuestos a $60^{\circ}$ y $120^{\circ}$ respectivamente. También ambas placas tienen acopladas tres esferas de caracterización, las cuales son utilizadas para determinar los sistemas de coordenadas de cada una de las placas. Estas esferas de caracterización son de gran importancia en la calibración de la plataforma y durante el uso de la plataforma en los procedimientos de verificación.

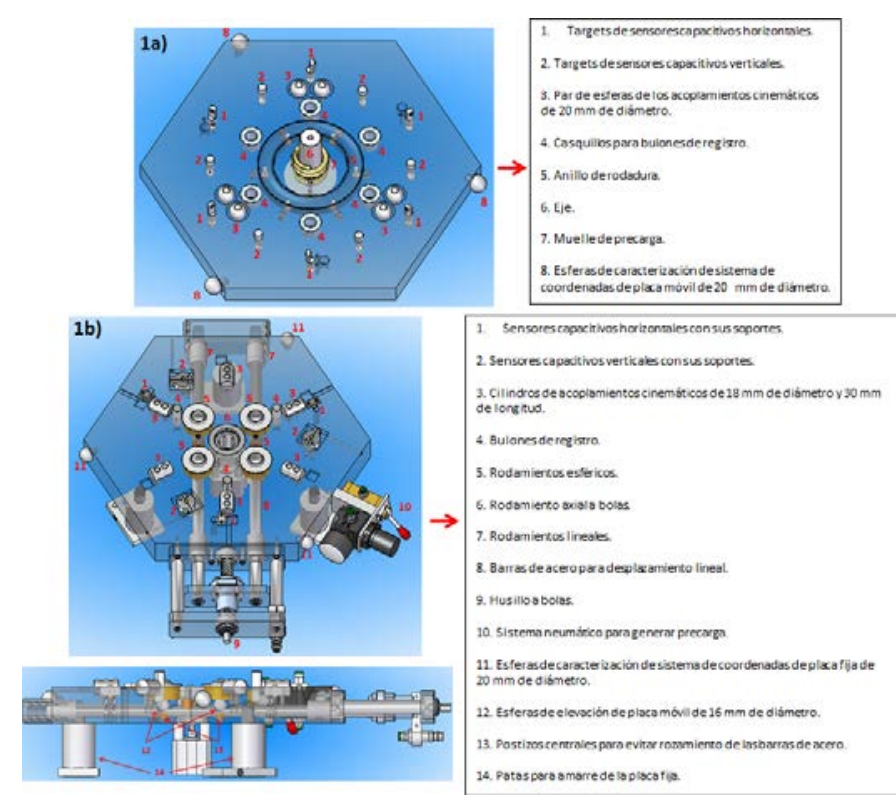


Figura la y $1 b$. Componentes mecánicos de la platafroma multi-registro.

En la figura 1a y 1b se muestran los componentes mecánicos más importantes que conforman la placa móvil y placa fija respectivamente.

\section{Mecanismo de elevación}

El sistema de elevación mecánico consiste en un husillo a bolas, dos barras de acero con movimiento horizontal y dentro de cada una de las barras, se alojan dos esferas de $13 \mathrm{~mm}$, las cuales se encuentran debajo de unos rodamientos esféricos sobresaliendo por un agujero cilíndrico en el centro del asiento de esos rodamientos como se ilustra en las figuras $2 \mathrm{a}$ y $2 \mathrm{~b}$. La separación de la placa móvil se logra al girar el husillo $180^{\circ}$ en sentido horario. Al girar el husillo se genera un desplazamiento lineal de las dos barras de acero que contienen las esferas de elevación, de manera que las esferas suben o bajan por la barra y el alojamiento cilíndrico, empujando el rodamiento de bolas y elevando o descendiendo la placa móvil.

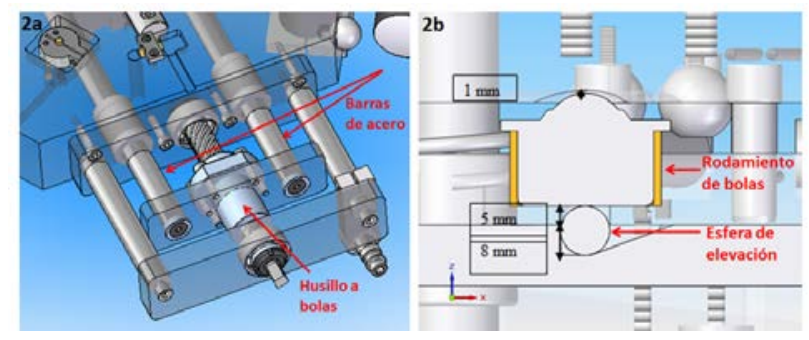

Fig 2. Ilustración de mecanismo de elevación.

\section{Mecanismo de rotación y descenso}

Una vez que la placa móvil se eleva por medio del sistema de elevación, ésta puede rotar alrededor de su eje central. Esto se consigue por medio de los rodamientos esféricos, los cuales son guiados por un anillo en forma de rodadura (figura $3 \mathrm{~b}$ ) que asegura un movimiento rotacional. Debido a que los acoplamientos cinemáticos son colocados cada $60^{\circ}$, la placa móvil se rotara $60^{\circ}$ para registrar una nueva posición, para posteriormente, descender acoplando perfectamente los acoplamientos cinemáticos.

Cuando la placa móvil se haya girado manualmente los $60^{\circ}$, el husillo se girará $180^{\circ}$ de regreso, lo que ocasionará que las barras de acero así como las esferas de elevación y los rodamientos esféricos regresen a su posición inicial, posicionando la placa móvil en posición cerrada como se muestra en la figura 3a. Para asegurarse que la placa móvil se ha girado $60^{\circ}$, se agregan 3 bulones de registro atornillados a la placa fija con sus respectivos alojamientos en la placa móvil. Cuando la placa móvil desciende, los bulones de registro deberán quedar dentro de los alojamientos, ya que en caso de no ser así, la rotación no sería exactamente de $60^{\circ}$ por lo que se tendría que subir de nuevo la placa móvil y completar la rotación hasta que los bulones de registro encajen perfectamente en los alojamientos como se puede observar en la figura 3c.

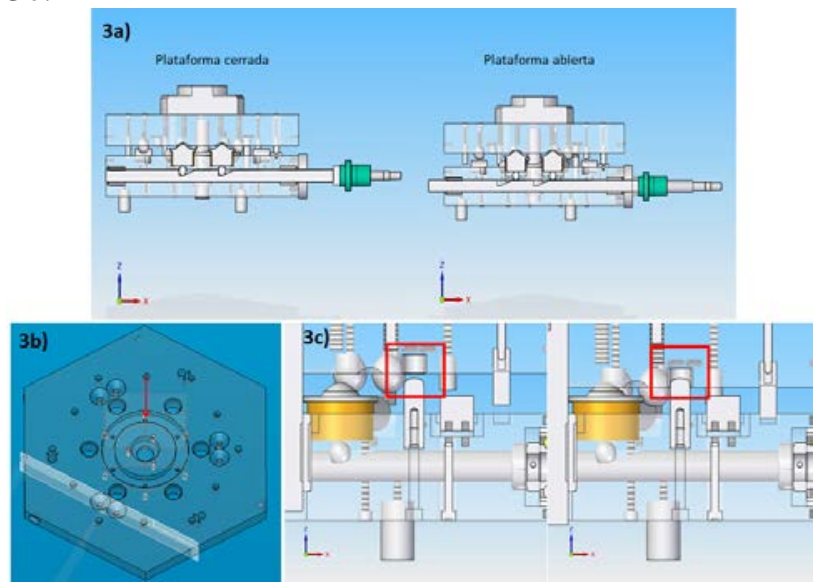

Figura 3. Mecansimos de rotación y descenso.

\section{Conclusiones}

En este trabajo se muestran el uso de los componentes mecánicos que conforman la plataforma y el funcionamiento de cada uno de los mecanismos que permiten el funcionamiento correcto de la plataforma para poder ser utilizada en los procedimientos de y verificación de los equipos portátiles.

\section{Agradecimientos}

Agradecimiento del primer autor a la Dirección General de Educación Superior Tecnológica (DGEST) y el Consejo Nacional de Ciencia y Tecnología (CONACYT).

\section{REFERENCIAS}

[1] “ASME B89.4.22: Performance Evaluation of Articulated Arm Coordinate Measuring Machines,” 2004.

[2] "VDI-VDE 2617-9: Accuracy of Coordinate Measuring Machines; Characteristics and their Reverification,” 2009. 